\title{
Humanitarian logistics and cultural diversity within crowd simulation
}

\author{
Alberto Ochoa ${ }^{1}$, Isaac Rudomin ${ }^{2}$, Genoveva Vargas-Solar ${ }^{3,4,5}$, Javier A. Espinosa-Oviedo ${ }^{2,5}$ \\ Hugo Pérez ${ }^{2}$, José-Luis Zechinelli-Martini, \\ 1 Universidad Autónoma de Ciudad Juárez, México \\ 2 Barcelona Supercomputing Centre \\ ${ }^{3}$ French Council of Scientific Research, \\ ${ }^{4}$ Laboratory of Informatics of Grenoble \\ ${ }^{5}$ French Mexican Laboratory of Informatics and Automatic Control \\ ${ }^{6}$ Fundación Universidad de las Américas, Puebla \\ alberto.ochoa@uacj.mx, isaac.rudomin@bsc.es,genoveva.vargas@imag.fr \\ javiera.espinosa@bsc.es, hugo.perez@upc.es, joseluis.zechinelli@udlap.mx
}

\begin{abstract}
Human stampedes caused by panic and overcrowding occur frequently as a result of abnormal events (e.g., a fire or explosion) produced in collective events (e.g., religious pilgrimages, music concerts and sportive events). These events provoke panic and when people are agglomerated they try to escape pushing each other without realizing that others are being crushed. Since crowds can consist of individuals with diverse physical and social characteristics determined by cultural diversity, it is difficult to configure the space in advance and find solutions in real-time to save people and reduce catastrophe. This paper proposes an approach to explore the impact of anthropometry and cultural diversity in the behaviour of crowds in panic situations. Our approach includes techniques for reproducing and simulating the behaviour of the crowd to generate models that can help decision making to control such situations. The main contribution of our work is to use computational science, data processing and visualization techniques to perform our simulation and study, for eventually supporting critical decision making.
\end{abstract}

Keywords. crowd simulation, efficient data management, multi-agent systems.

\section{Logística humanitaria y diversidad cultural en la simulación de multitudes}

Resumen. Las estampidas humanas, causadas por el pánico o por la sobrepoblación, ocurren frecuentemente como resultado de eventos anormales (por ej., un incendio o una explosión) producidos en eventos colectivos (por ej., peregrinaciones religiosas, conciertos musicales y eventos deportivos). Estos eventos crean pánico provocando a las personas hacinadas a tratar de escapar empujándose sin darse cuenta que otras personas están siendo atropelladas. Dado que las multitudes pueden consistir en individuos con características físicas (i.e. antropométricas) y culturales diferentes determinadas por su diversidad cultural, es difícil prever configurar el espacio y encontrar soluciones en tiempo real para salvar a las personas y reducir la posibilidad de catástrofes. Este artículo propone una estrategia para explorar el impacto de la diversidad cultural en el comportamiento de multitudes en situaciones de pánico. Nuestra estrategia incluye técnicas para reproducir y simular el comportamiento de las multitudes que permita generar modelos que puedan ayudar a la toma de decisiones para controlar esas situaciones. La contribución principal de nuestro trabajo es el uso de técnicas de ciencia computacional, procesamiento de datos y visualización para efectuar nuestro estudio y simulación para apoyar, eventualmente, la toma de decisiones críticas.

Palabras clave. Simulación de multitudes, administración de datos eficiente, sistemas multi-agentes.

\section{Introduction}

Human stampedes caused by panic and overcrowding occur frequently as a result of abnormal events (e.g., a fire or explosion) produced in collective events (e.g., religious pilgrimages, music 
concerts and sportive events). These events provoke panic and when people are agglomerated they try to escape pushing each other without realizing that others are being crushed. Since crowds can consist of individuals with diverse physical (i.e., anthropometric) and social characteristics determined by cultural diversity, it is difficult to configure the space in advance and find solutions in real-time to save people and reduce catastrophe.

Crowd simulations for humanitarian logistics provide means for studying crowd behaviour under different conditions. After studying video pedestrian patterns, behavioural scientists have concentrated on the rules that people are accustomed to using in their daily lives and transform them into heuristic formulas. They have focused mainly on four types of danger associated with crowds: (i) trampling and crushing at religious sites; (ii) trampling, crushing and drowning on ships at sea or waterways; (iii) crushing in massive concerts or enclosed spaces such as clubs; (iv) contingency situations of nature, as can be earthquakes, floods, avalanches or landslides, destruction of human made structures.

Simulating crowded places helps architects to design safe public spaces. However, until now, they have ignored everyday human behaviour when walking, particularly in groups, waiting for children or elderly members of the group, or stopping to talk, or even tying shoelaces, which imply much more complex simulations. Indeed, humans react to stress and crowding in different ways depending on their size, age, gender and other physical characteristics; on their personal psychological characteristics; on whether they are alone or in groups such as family. Their behaviour also changes according to culture dependent characteristics such as proxemics, and even by how people of different cultures understand instructions. Ignoring these fundamental aspects dehumanizes people by simply considering them as particles that react to forces according to their mass.

This paper proposes an approach to explore the impact of anthropometry and cultural diversity in the behaviour of crowds in panic situations. Human density makes people come into physical contact creating behaviour that can be understood as an exchange of real physical forces. Our approach models crowds using microscopic simulation using multi-agent systems, where dynamics is coupled with crowd simulation modified by anthropometric and cultural parameters. Our approach includes techniques for reproducing and simulating the behaviour of the crowd to generate models that can help decision making to control such situations. The main contribution of our work is to use computational science, data processing and visualization techniques to perform our simulation for eventually supporting critical decision making.

The remainder of the paper is organized as follows. Section 2 presents a general overview of existing work dealing with challenges related to crowd studies focusing on behaviour modelling, simulation and decision making. We particularly address data management issues related to the analysis and simulation processes of the crowds in the context of humanitarian logistics. Section 3 introduces our agent based approach for simulating crowds for addressing human logistics. Section 4 describes the experimental validation that we conducted for simulating a case of stampede and discusses our results. Section 5 concludes the paper and discusses future work.

\section{Related work}

Pedestrians have been empirically studied for more than four decades [1]-[3]. Initial methods were based on direct observation, photographs, and time-lapse films to develop a level-of-service concept [4], design elements of pedestrian facilities [5]-[8], or planning guidelines [9]. Most research into panic, however, has been of empirical nature (e.g., [10]-[13]), carried out by social psychologists and others.

Simulation models have been proposed for addressing crowd dynamics modelling, route choice behaviour of pedestrians, and emergency and evacuation situations. Experimental efforts have revealed quantitative details of pedestrian interactions, which have been modelled by mathematical equations. Corresponding computer simulations of large numbers of pedestrians have been compared with the empirically observed dynamics of crowds. Such studies have led to a deeper understanding of how collective behaviour 
on a macroscopic scale emerges from individual human interactions. The analysis of crowds using mathematical and simulations lead to important conclusions for developing human logistics strategies. For example, self-organized patterns of motion demonstrate that "intelligent" collective dynamics can be based on simple local interactions; under extreme conditions, coordination may break down giving rise to critical crowd condition such as the so called "freezing-by-heating" and "faster-is-slower" effects, but also the transition to "turbulent" crowd dynamics. This Section describes existing works according to four perspectives: techniques used for observing high density crowds which we understand as possible data harvesting methods (Section 2.1); crowd models that provide mathematic abstractions of crowd behaviour (Section 2.2); simulation of crowds that apply mathematical and artificial intelligence methods for reproducing the way crowds evolve under different conditions (Section 2.3); finally humanitarian logistics that integrate existing methods into systems that support decision making (Section 2.4).

\subsection{Observing high density crowds}

The computer vision community has started taking interest in addressing different research problems related to the scenarios involving large crowds of people. Existing approaches focus on the tasks of crowd detection, and detection and tracking of individuals in the crowd. These approaches have mainly used representations based on $x t$ slices of spatio-temporal video volume [2], shape and colour models of individuals [14], boundary contours [15], trajectories of interest points [16], and approaches which explicitly count the number of people in the crowd [2], [3].

Computer vision algorithms cannot handle crowded scenes because their performance concerning object detection, tracking and event detection decreases in such conditions. [17] addresses this problem by modelling moving crowds as aperiodic dynamical systems manifested by a time dependent flow field. Such a flow field in a general scene consists of regions with qualitatively different dynamics, reflecting the motion patterns emerging from the spatio-temporal interac- tions of the participants between each other and with the physical world.

[18] also proposes a crowd classification with respect to density and then it discusses methods that can be applied to track people within crowds. In a scene with a sparse or moderate crowd, most pedestrians can be fully observed and the pedestrians can be detected with a frame-based human detector. Persons are then tracked by combining detections into tracklets and associating the tracklets into long trajectories [5], [19].

[20]-[22] propose the Data Association based Tracking (DAT) algorithm which is extended by using shape and appearance models [23], bodypart detectors [24], or a boosting algorithm to train the parameters [25]. However, in a denser crowd where the pedestrians are heavily occluded by the others, frame-based detection is highly unstable, and associating among a large number of trajectories is very expensive. To address this limitation, it is possible to use local feature points to track individual within a crowd. Brostow et al. [16], Li et al. [26], and Sugimura et al. [27] were among the first to do this. They assume that the feature points belonging to the same person are close in space and their motion exhibit high correlation over time. The most important limitation of this approach is that targets moving together with the same speed cannot be identified as separate persons, whereas a local body movement is usually wrongly estimated as a different target.

Optical flow algorithms have been widely used for tracking pedestrians. Initial approaches assume that optical flow on the target is uniform, and the target is tracked by computing the mean flow around the target location [28], [29]. Some approaches use foreground-background segmentation to get a precise target region. In crowded scenes, targets are frequently occluded and their region changes over time. As a result, the target location does not change linearly. This requires a propagation model that adaptively changes over time and space with non-linear behaviour like the solutions proposed by Rodriguez [15] and Kratz [30]. In the high density and the extremely dense crowd situations, the most promising tracking algorithms use motion information in local areas. For example, Ali and Shah [17] assume that pedestrians in the crowd behave as particles in the flow. This assumption leads to a solution only 
able to track pedestrians moving in the same direction as the crowd. Rodriguez et al. [31] first divide the video into short clips and for each local area in the clip, flow vectors are quantized into four categories based on the direction where vectors are heading. The quantized clips are trained within the Correlated Topic Model (CTM), generating a set of topics. For each new frame, a probability distribution over the topics is measured, and the probability of the motion is then derived. The new target position is estimated as a combination of the observation and the tracker prediction.

\subsection{Modelling crowds}

In his fruitful work Helbing has proposed a simplified model of pedestrian interactions: the "social force model". He has addressed crowd dynamics, typically the formation of large-scale spatiotemporal patterns of motion, when many pedestrians interact with each other. Helbing et al. [32] found that high-density flows, such as flows with extreme local densities of up to 10 people per square meter, can result in a phenomenon called crowd turbulence and can trigger the trampling of pedestrians. To prevent such disasters highdensity flows must be avoided, particularly the three conditions that can result in a disaster: (i) low infrastructure capacity, (ii) large numbers of pilgrims, and (iii) merging flows, intersecting flows, or counter flows (i.e., flows of people moving in opposite directions), especially in reduced spaces. If many pedestrians head toward or pass through a location with low infrastructure capacity in a short time, this location can become a bottleneck with high densities and a high risk of crowd turbulence.

\subsection{Simulating crowds}

Numerous occurrences of stampedes in the recent past have shown that better coordination between public safety organizations and remodelling alone cannot solve the problem of managing large crowds. Prediction of pedestrian-flows in pedestrian zones, and buildings with an exceptional architecture or in challenging evacuation situations, have been addressed by simulation models (e.g. queuing models [33], transition ma- trix models [34], and stochastic models [35]). In addition, there are models for the route choice behaviour of pedestrians [7], [36]. Helbing observed that none of these modes adequately takes into account the self-organization effects occurring in pedestrian crowds. A first modelling approach that seems suited to reproduce spatiotemporal patterns of motion was proposed by Henderson [37], [38], who conjectured that pedestrian crowds behave similar to gases or fluids. Realistic gas-kinetic or fluid-dynamic theory for pedestrians contain corrections due to their particular interactions (i.e. avoidance and deceleration manoeuvres). Thus, for practical applications a direct simulation of individual pedestrian motion is easier and more flexible to achieve. In consequence, pedestrian simulation mainly focuses on agent-based models of pedestrian crowds, which allow to consider local coordination problems. The "social force model" [31], [39] is maybe the most well-known of these models, but there are also cellular automata of pedestrian dynamics models [6], [8], [40]-[44] and Al-based models [45], [46]. Below we present a brief description of the main behavior models based on Al.

Reynolds [47] proposed one of the first solutions for large groups of entities with emergent behaviour being an extension of particle systems. It is based on three basic rules: separation, alignment, and cohesion. These rules maintain together, in a direction and free of collisions a group of boids or bird-like objects.

The Predictive/Velocity-based models [48] propose methods where agents compute the set of velocities that lead to a collision with an obstacle. To move on routes without collision, agents choose velocities outside this domain. This concept is expanded by Van den Berg [49], [50], introducing the notions of Velocity Obstacles (VO) and Reciprocal Velocity Obstacles (RVO) and the notion of Optimal Reciprocal Collision Avoidance (ORCA).

In the everyday exercise of controlling their locomotion, human beings rely on the visual information obtained from the perception of the environment to achieve collision-free navigation. Cognitive science allows extracting relatively succinct information from perception to reach a safe locomotion, e.g., using the distance of obstructions in candidate lines of sight, pedestrians apply simple 
heuristics to adapt their walking speeds and directions. Synthetic Vision models

When observed, the individuals in a crowd seem to adopt an "automatic behaviour" that can be interpreted as the result of a learning process based on trial and error [32], which has been simulated with evolutionary algorithms [51]. For example, pedestrians have a preferred side of walking since an asymmetrical avoidance behaviour turns out to be profitable [32], [39]. [25], [32] claim that the formation of a behavioural convention can be described by means of evolutionary game theory. Based on quantitative measurements studies have shown that the behaviour in conflict situations can be described by a superposition of forces [52], [53]. In this sense, Lewin [54] proposes a mathematical model based on the idea that behavioural changes are guided by social fields or social forces [39], [55].

\subsection{Humanitarian logistics}

Humanitarian logistics specializes in organizing supplies during natural disasters or complex emergencies to the affected area and people. Technology is a key factor to achieve better results. Implementing up-to-date information or tracking systems and using humanitarian logistics software can provide real-time supply chain information, organizations can enhance decision making, increase the quickness of the relief operations and achieve better coordination of the relief effort. Existing (humanitarian) logistics systems are operations research based decision support systems (ORDSS) for crowd management. ORDSS employ a range of tools from operations research, analytics, and crowd dynamics [56]. At its core, such a system implements a scheduling tool and a real-time video tracking system. The video tracking system measures infrastructure utilization, and an integrated series of mixedinteger programs and quadratic programs balance capacity utilization by considering preferred stoning times and infrastructure capacities. The ORDSS provides solutions that enable uncongested and smooth pilgrim flows, for example, and extensive real-time reporting.

\section{A sociocultural perspective for simulating human stampedes}

Unlike existing contributions, in this research we focus on sociocultural aspects identified in the last stampedes occurred in Mecca, and demonstrate their impact in a possible humanitarian catastrophe for example in the holy places of Islam. Figure 1 gives an overview of our approach for simulating stampedes taking into consideration sociocultural and anthropometric perspective.

The principle of our approach is to develop a multi-agent system for simulating the behaviour of a dynamic crowd consisting of multicultural individuals evolving in an urban space.

- An urban space is a spatio-temporal region modelling an "urban" configuration consisting of a geometric area with signals exists designed for dynamic pedestrians' flows of specific densities moving according to predefined trajectories. Spaces configurations and characteristics are designed considering an anthropometric model.

- The anthropometric model describes the physical characteristics of people that will visit that urban space.

- The organization of the space, the signals and amenities serving to control the crowd in normal and abnormal situations must consider sociocultural aspects.

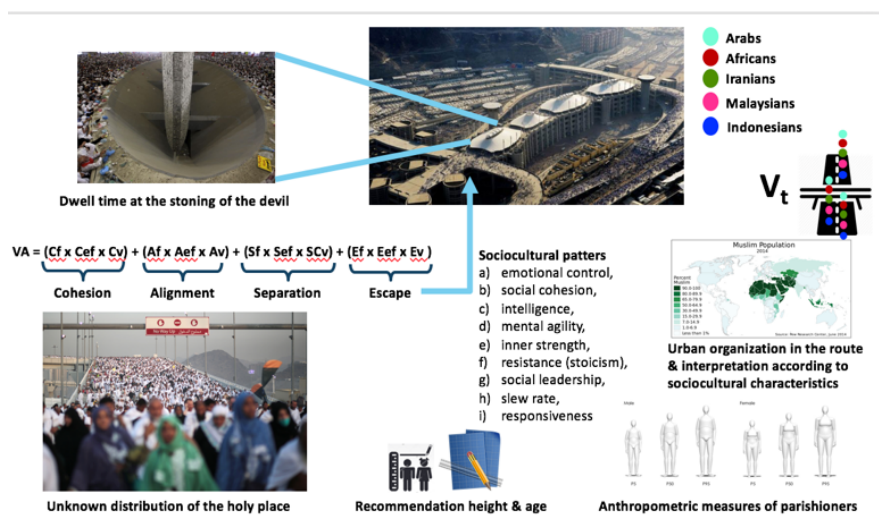

Figure 1. Simulating and studying stampedes from a sociocultural and anthropometric perspective

These aspects are included in the behaviour model associated to the agents of our system, 
that will behave according to a social behaviour implemented by a mathematical model with associated rules and constraints representing anthropometric and sociocultural models to make the behaviour of agents as realistic as possible. The objective being to simulated a dynamic pilgrim crowd as close as possible to reality.

\subsection{Anthropometric and sociocultural model}

There is variability between the body dimensions of different people, because of their age, gender and ethnicity. This variability makes it necessary to measure the population of people who will use the facilities to host a dynamic crowd for example in a pilgrimage. The objective is to design the spatial area and the trajectory according to the range of measures of each person belonging to the crowd. For this, measures should be expressed for a specific configuration of pilgrims according to their size and phenotypic values associated with standard deviation and percentiles of that population. The phenotypic values and anthropometric measures are given by existing models that we adopt (see Table 1).

Another important aspect to consider particularly for logistics and organization reasons, both in normal and abnormal situations, are sociocultural aspects. For example, in one of the human stampedes in Mecca, two leading forces were identified by analysing videos. Iranians whose average height often exceed 1.89 and greater muscle mass, and Malians with similar anthropometry but with different languages and sociocultural references. Analysts observed the lack of information in different languages, but also a sociocultural aspect of group formation when walking particular in Iranians and parishioners from Africa. This behaviour is not present in the Saudi Arabian society. It is important to see how different groups react to visual signs, since in some cultures visual warnings are not used, and inexperienced travellers, both adults and children, can get confused and unable to follow instructions for proper evacuation. Therefore, in our model we considered sociocultural aspects of the individuals participating in crowds. Since we are particularly interested in simulating dynamic crowds, pilgrimages with years of tradition, we assume that we know the average distribution of nationalities attending, the average distribution of men, women and children, and we also know out of ethnographic and crowd tracking studies, the cultural aspects that can have impact on the behaviour of the crowd in the presence of an abnormal event, e.g., stampede.

The sociocultural aspects that we considered in our model include: collective behaviour in public spaces (civility, awareness of traffic signs, respectfulness of traffic signs), automatic behaviour in dynamic crowds that follow a specific direction (whether people tend to form lines, lineage to the right/left) and the vital space (the minimum and maximum separation space between two people). For individuals' social behaviour when an abnormal event appears, we adopt the assumptions of existing work saying that crushing and pushing depends on the urban facilities of the space and of a kind of irrational behaviour of the crowd that depends on the global vision or knowledge that people can have of the space. This knowledge can create or not confidence and reduce the degree of panic.

\begin{tabular}{l|l|l|l}
\hline Variables & Measurement Unit & Variables & Measurement Unit \\
\hline Age & Years & Height & Centimeters \\
\hline Weight & Kilograms & Body Mass Index (BMI) & $\left(\mathrm{kg} / \mathrm{m}^{2}\right.$ \\
\hline Total Arm Length & Centimeters & Upper Arm Length & Centimeters \\
\hline Lower Arm Length & Centimeters & Wrist Circumference & Centimeters \\
\hline Upper Arm Circumference (REST) & Centimeters & Upper Arm Circumference (contracted) & Centimeters \\
\hline Chest Circumference & Centimeters & Chest Circumference (Elevated) & Centimeters \\
\hline Hip Circumference & Centimeters & Thigh Circumference & Centimeters \\
\hline Calf Circumference & Centimeters & Hand Width & Centimeters \\
\hline Shoulder Width & Millimeters & Hip Width & Millimeters \\
\hline Bi-Humerus Diameter & Millimeters & Bi-femur Diameter & Millimeters \\
\hline Wrist Diameter & Millimeters & Body Density (BD) & (gm/cc) \\
\hline Percentage Body Fat & \% & Total Body Fat (TBF) & Kilograms \\
\hline Lean Body Mass (LBM) & Kilograms & Grip Strength (Rigth Hand) & Kilograms \\
\hline Grip Strength (Left Hand) & Kilograms & & \\
\hline & & & \\
\hline
\end{tabular}

Table 1. Anthropometric measurements considered in our model

We designed our agent system taking into consideration the anthropometric model and sociocultural knowledge. This guides the behaviour of the agents when they evolve in the virtual space and we thereby obtain a realistic simulation of the dynamic crowd. Indeed, avatars representing agents in the crowd have anthropometric characteristics considered in our model. Their interaction with the environment and with other agents is guided by the associated sociocultural constraints. Section 4 shows how these attributes are interpreted into concrete measurements implemented by agents used for simulating a concrete dynamic crowd. 


\subsection{Crowd social behaviour observatory}

Figure 2 presents the general functional architecture of a crowd social behaviour observatory. It is a system with three main components: the data harvesting one, which provides mechanisms for collecting data stemming from the environments in which crowds evolve. The data can be related to the trajectories of people, videos, the spatial configuration of the space, the urban organization, facilities, the adopted anthropometric and sociocultural models. It can also include harvesting of data stemming from social networks (services in Figure 2).

The objective is to acquire a close real-time vision of dynamic crowds evolving in predefined spaces with well-known urban organization and facilities. In the case of crowds like the one we analysed in our experimentation (pilgrimage) this is technologically possible since for example the individuals participating in the pilgrimage will have a digital bracelet that will produce data about their behaviour. We apply moving objects database techniques coupled with continuous spatiotemporal queries. Collected data can be queried on-line. Table 2 shows the taxonomy of queries that we can perform on collected data. The taxonomy considers continuous and batch data production/consumption, and nomad and static data consumers and producers.

As shown in table 2 it is possible to ask for the closest emergency exits from "my" current position, those that are in a region of 50 metres. These queries can be asked on top of the data collected from the environment. For moving consumers and producers these queries should be executed recurrently since the validity of the results change in time. These queries can be executed locally by the agents simulating the crowd, by individual participating in a collective event where they are part of a dynamic crowd through a Web application, and by the staff piloting the logistics of the collective event. Representative datasets are continuously integrated and updated to provide new input to the agents of the simulation that reproduce the behaviour of the dynamic crowd which is visualized in a 3D virtual setting (rightmost side of Figure 2).

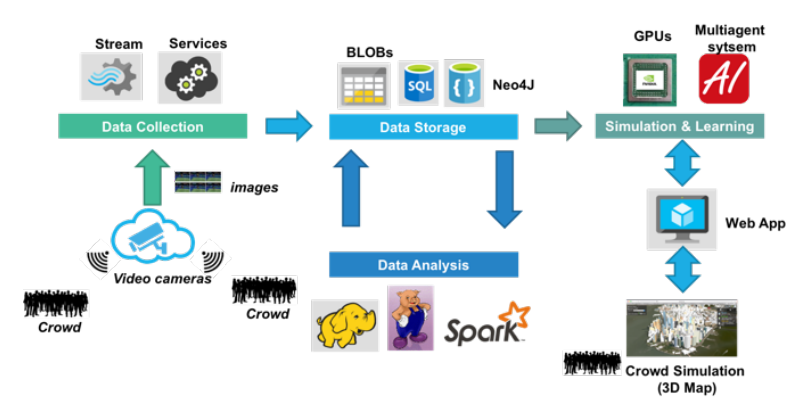

Figure 2. General architecture

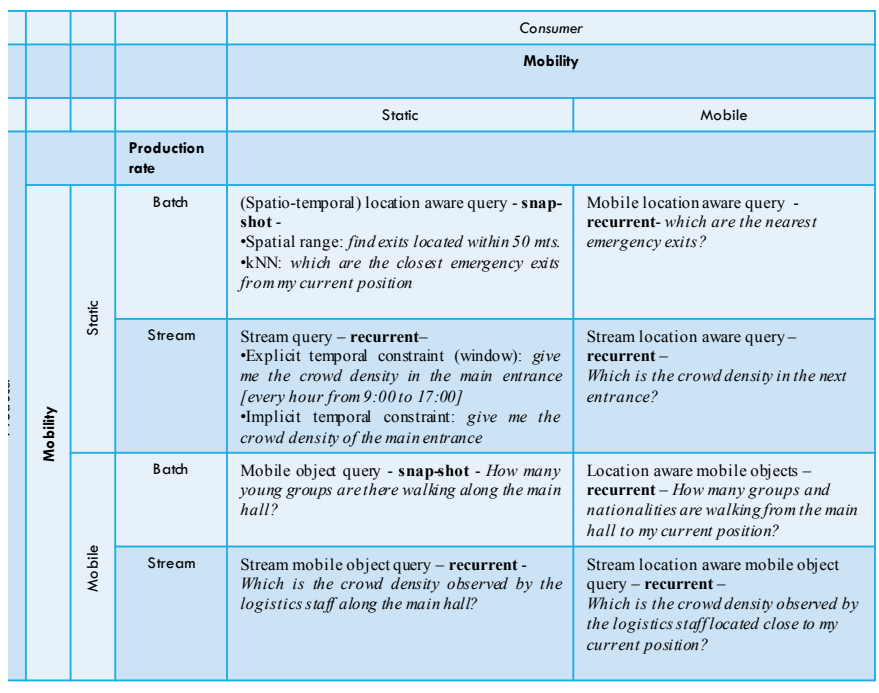

Table 2. Query taxonomy

Figure 3 shows the general architecture of an agent that composes the multi-agent system that simulates a dynamic crowd in our approach. An agent is a complex autonomic intelligent system that implements an event-reaction cycle guided by the internal knowledge it computes thanks to the data it receives and processes. The data are produced:

- Continuously: the events happening in the environment through the Perception module.

- In batch: concerning the general plan of the space where the agent will move. These data include the objects in the space, the urban facilities, and they are store in the World model base of the knowledge module. 


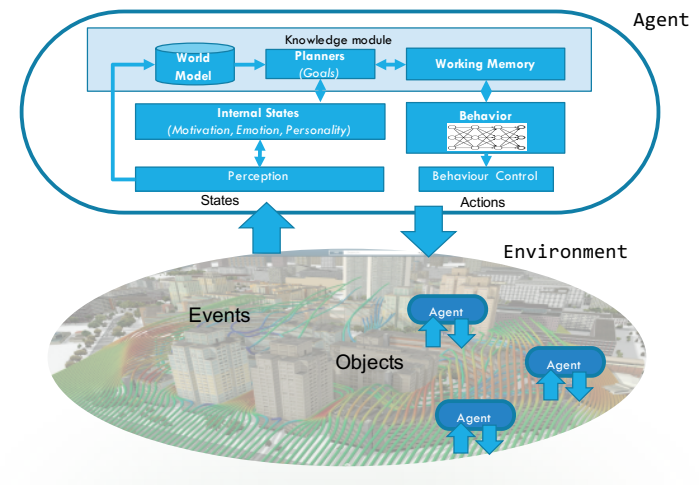

Figure 3. General architecture of an agent

The agent processes these data guided by the behaviour rules coded the Behaviour module. The agent plans goals and executes actions that will have impact on the environment.

The distributed knowledge is used by the multi-agent system that generates a collective behaviour. This behaviour is implemented in the simulation of a dynamic crowd evolving in a collective event. The agents and environment of our approach have a projection in a virtual 3D world that visualizes their individual and collective behaviour.

\section{Experimentation}

Our experiment is related with a religious event in a Muslim world with people from diverse societies, heterogeneous sociocultural characteristics (e.g., different nationalities and having different understanding of the visual announcements written in Arab) and anthropometry.

We chose Menge ${ }^{1}$, a cross-platform, extensible, modular framework for simulating pedestrian movement in a crowd, including social behaviours related with paranoia. Menge's architecture is inspired by an implicit decomposition of the problem of simulating crowds into four components: religious masses, mass concerts, shipwreck and floods. Different combinations of subproblem solutions yield crowd simulators with likewise varying properties. Menge creates abstractions for those subproblems and provides a plug-in archi-

\footnotetext{
${ }^{1}$ http://gamma.cs.unc.edu/Menge/
}

tecture so that a novel simulator can be dynamically configured by connecting built-in and bespoke implementations of solutions to the various subproblems. We used Unity ${ }^{2}$ a cross-platform game engine for implementing the visualization of the dynamic crowd of the Hajj.

\subsection{Use case: the Hajj}

The Hajj, the annual Muslim pilgrimage to Makkah in Saudi Arabia, is one of the largest pedestrian events in the world. It takes place from the 8th to the 13th day of Dhu al-Hijjah, the 12th and last month of the Islamic (lunar) calendar. Each year, up to four million pilgrims approach the holy sites in the region of Makkah to perform their religious duty during many days. In 1950, fewer than 100,000 pilgrims performed the Hajj; in 2005, the number of pilgrims performing it exceeded two million for the first time. Until 2006, several crowdrelated disasters led to thousands of casualties. Thus, the Ministry of Municipal and Rural Affairs of the Kingdom of Saudi Arabia (MOMRA) launched projects to prevent future crowd-related accidents.

\subsection{Experiment design}

To simplify the simulation problem, in the design of the experiment (DOE) we consider that the crowd attending the Hajj is composed of groups belonging to four kind of Muslim societies: Iranians, Muslim Africans, Muslims Asians no Arabs and Arabs. To obtain an accurate model and social behaviour of the crowd our system maintains data about representative individuals in each group and data related to the cultural aspects. The latter is done in order to distribute in an optimal way for each zone of the mosque associated to a group a specific social behaviour. We propose a realistic model using Unity to visualize the diversity of individuals of different kinds of social groups (See Figure 4).

The main experiment consisted in evaluating individuals from group of parishioners, and performing a simulation on 200 replicas for 100 instances of individuals. This allowed the genera-

\footnotetext{
${ }^{2}$ https://unity3d.com
} 
tion of the best selection of every social group and its possible location in the representation of the Design of Experiment (see Figures 5 and 6). The location of each group is obtained by comparing the different cultural and social similarities of each group, and evaluating the proposed multiple matching model.

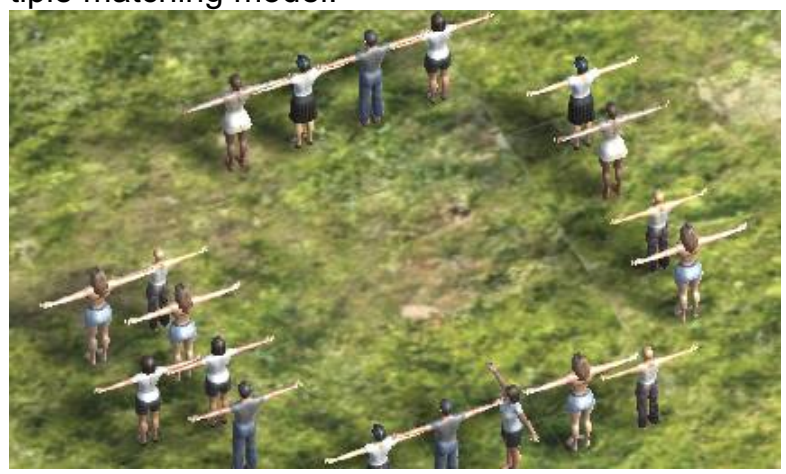

Figure 4. Visualization of individuals of different kinds of social groups in the Hajj event

The weight vector used for the fitness function is:

$$
\begin{aligned}
& \mathrm{Wi}=[0.6,0.7,0.8,0.5, \\
& 0.6,0.4,0.9,0.5,0.4]
\end{aligned}
$$

representing respectively, the importance of particular attributes associated with a good parishioner (see Table 3): a) emotional control, b) social cohesion, c) intelligence, d) mental agility, e) inner strength, f) resistance (stoicism), g) social leadership, h) slew rate, i) responsiveness.

Each attribute is represented by a discrete value between 0 to 5 , where 0 represents the null value and 5 the highest value of the attribute. The DOE selects the colour of each group based on the similarity of these attributes. Similarity is determined as follows. The DOE tests the orthogonal matrix with interactions among variables studied within a colour range (1 to 64). The orthogonal matrix is $\mathrm{L}-\mathrm{N}\left(2^{8}\right)$, eight times the $\mathrm{N}$ executions. The value of $\mathrm{N}$ is given by the combination of the 8 possible values of the variables, also the values of the colour palette.

Table 3 shows some possible scenarios as a result of the combination of the values of the attributes and specific sociocultural to represent a problem in a religious place (meaningful representation to participate in the Hajj).

The use of orthogonal matrix test facilitates the reorganization of the different attributes of a parishioner. It also helps to specify the best chance to correct and identify appropriate skills to perform the Hajj, for each selected group.

\begin{tabular}{|c|c|c|c|c|c|c|c|c|}
\hline $\mathbf{a}$ & $\mathbf{b}$ & $\mathbf{c}$ & $\mathbf{d}$ & $\mathbf{e}$ & $\mathbf{f}$ & $\mathbf{g}$ & $\mathbf{h}$ & $\mathbf{i}$ \\
\hline 0 & 1 & 2 & 2 & 3 & 3 & 4 & 5 & 1 \\
\hline 0 & 1 & 2 & 2 & 3 & 4 & 5 & 5 & 1 \\
\hline 1 & 1 & 3 & 2 & 4 & 4 & 2 & 1 & 2 \\
\hline 1 & 1 & 3 & 2 & 5 & 3 & 2 & 1 & 2 \\
\hline
\end{tabular}

Table 3. Orthogonal matrix for characterizing the Hajj population

\subsection{Simulation results}

The results allow us to analyse the effect of the variables in the colour selection of all possible combinations of values reported in Table 2. The colour code corresponds to the one discussed in the previous Section: Iranians in green, Arabs in Cyan, Blue and Rose respectively Indonesians and Malaysians, Africans in red. Figure 5 shows the distribution of agents generated for each group according to their social cohesion, anthropometric and sociocultural characteristics. It shows that we generated a representative population that we can use to simulate their behaviour during the Hajj event according to the characteristics of the space in which they evolve.

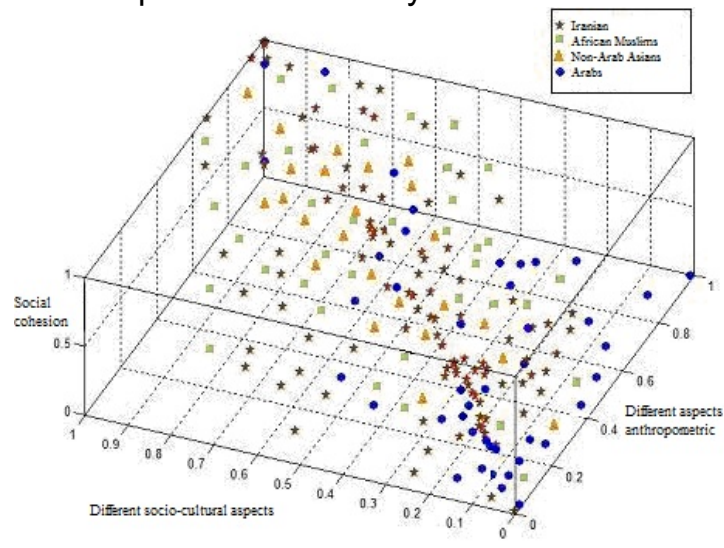

Figure 5. Spatial representation of sociocultural behaviour categories of the four types of participants in the religious Hajj event 
Since we simulated the behaviour of pilgrims during the Hajj circuit they perform in the Masjid al-Haram mosque, we represented the mosque distribution plan. In order to understand the distribution, we first explain the way the ritual of the pilgrims is organized in the mosque. The ritual of Tawaf that they perform includes: (1) walking seven times counter clockwise around the Kaaba. During this circuit a pilgrim "stops" in front of the Black Stone or at least reduces her pace to point it. A pilgrim performs three rounds in a hurried pace and four in a leisure pace. Tawaf is now also performed on the first floor and roof of the mosque because of the large crowds. (2) Then, a pilgrim does two Rakaat prayers at the Place of Abraham a site near the Kaaba inside the mosque. Because of large crowds during the days of Hajj, they may instead pray anywhere in the mosque. (3) After prayer, pilgrims drink water from the Zamzam well, made available in coolers throughout the Mosque. (4) Finally, a pilgrim runs or walks seven times between the hills of Safa and Marwah, located near the Kaaba. The place is entirely enclosed by the mosque, and can be accessed via air-conditioned tunnels.

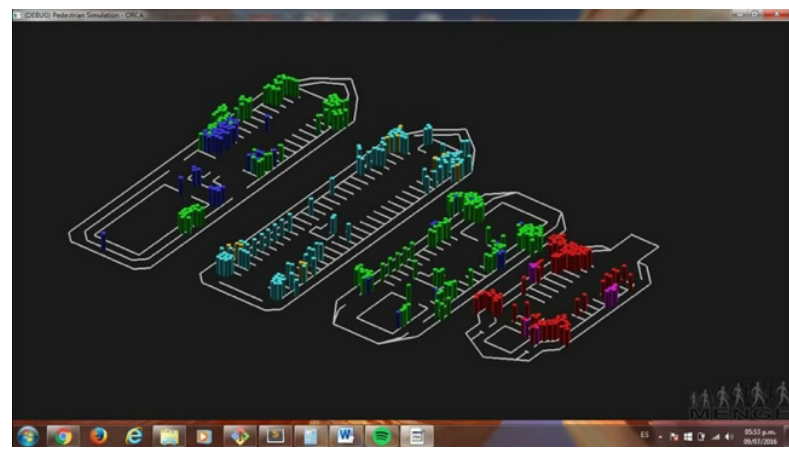

Figure 6. Distribution of different groups in the Masjid al-Haram mosque during the Hajj

Figure 6 shows the plan of the mosque (different levels) and zones in which the ritual is performed. The size of the boxes represents the density in each group (the number of individuals per square meter). In our simulation this is represented by the height of the boxes. Note that in our DOE a high density represents the situation in which 6 individuals share 1 square metre. Depending on the phase of the ritual the groups are performing, some zones of the mosque are more crowded than others.

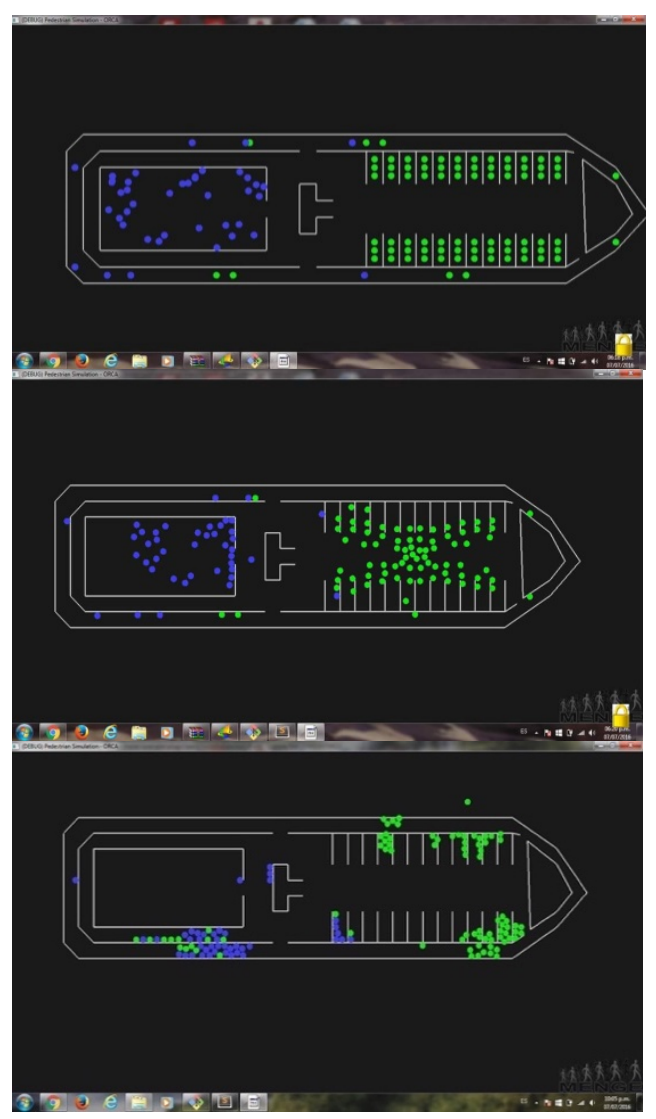

Figure 7. Simulation of pilgrimage evolution

As shown in Figure 6 we could simulate the groups participating in the Hajj in a realistic manner concerning: (i) the number of individuals in the crowd, (ii) the distribution of their anthropometric (coded in the colour of the box according to the colour code explained above) and sociocultural characteristics used to compute density which is visible in the size of the boxes in Figure 6 . Note that according to their group, pilgrims pray in different sections of the mosque. For example, Arabs in cyan are grouped together in the second level; Iranians are grouped in the first and third levels; and Indonesians, Malaysians and Africans mostly share the fourth level.

Then, it was important to model the dynamic aspect of the pilgrimage according to the predefined ritual performed through the spaces and 
tunnels of the mosque. In our current DOE we simulated a simplified version of the itinerary because for the time being we wanted the asses that our simulation could exhibit the "risky" places (e.g., tight tunnels and entrances).

Figure 7 shows the simulation results of the crowd trying to cross the different zones of the mosque. We show results of the first level which is occupied by Iranians (green points) and Indonesians (blue points). In the top of Figure 7 Iranians are in a kind of initial state and Indonesians are already moving in the surrounding tunnel and occupying the main "patio".

In a second moment, in the middle figure, the Iranian crowd starts to organize itself to gain one of the two exits and reach the tunnel. Since the flow has a direction in fact they can only use the right side exit. The middle and bottom of the Figure 7 sequence show how they organize to reach and go through the exit. First they go to the centre of the space then they gather themselves together in some point of the room while others approach the exit and start walking through the tunnel. At the same time, Indonesians (blue points) start moving towards the only exit in the right side of the square. Some gather themselves in the inner tunnel around the "patio" and the others in start going through the main tunnel.

We used this kind of sequences in our simulation to understand the collective behaviour of different groups. We were particularly interested in the way they behaved when they faced "delicate" facilities, for example tight entrances, or halls, tunnels or other important points where people tend to stop and the flow stalls. These critical aspects can help to determine the situations and possible humanitarian logistics problems to face in emergency situations and particularly avoid stampedes and crushes in a group that is larger than a million Muslim pilgrims.

Figure 8 shows the result of the simulation we conducted to measure the speed in which agents simulating pilgrims could run across the tunnels of the mosque. We decided to choose the last part of the itinerary where pilgrims have to go through the tunnels. To give a simple example, Figure 8 illustrates the case of Indonesian pilgrims. The objective of this simulation was to identify places where there could be potential crushes or stampedes.

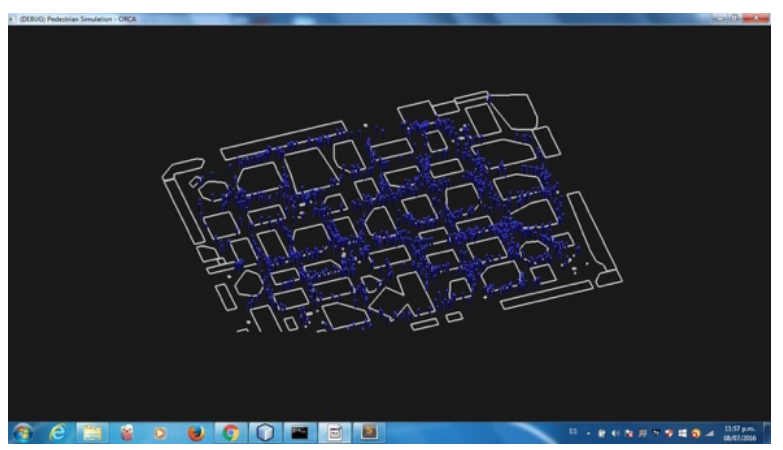

Figure 8. Detailed view of the distribution of pilgrims in the mosque during the Hajj

Note that the complex urban distribution of the mosque and the itinerary encouraging people to walk at different paces, and looking for spaces to accomplish all the phases of the ritual, creates potentially risky zones. People doing different phases of the ritual might find themselves going in opposite directions in tight tunnels. Other might want to access specific points without considering the density of the crowd and cause problems. Not respecting (or understanding) instructions, directions and signs can be fatal in such a complex layout. For the time being our work does not address specific recommendations, since this concerns our future work.

\section{Conclusions and future work}

This paper uses an agent based approach for modelling and simulating dynamic human crowds but it introduces the use of specific anthropometric and sociocultural aspects that have an impact on individual and group behavior. Different to existing models that focus on the flow dynamics of individuals in particular spaces and therefore provide a partial abstraction of individuals, our approach includes anthropometric and sociocultural aspects because: (i) we aim to address human logistics in which modelling both the urban space and the individuals of the crowds is important to predict and prevent catastrophes; (ii) we use virtual worlds to visualize the simulations of dynamic crowds in public spaces. In order to make virtual worlds realistic in big and fine grain it is important to reproduce avatar (i.e., agent) di- 
versity considering both their physical characteristics and their behaviour. The challenge is to reproduce realistic behaviours of the individuals interacting among each other and evolving in the space; but also of the social groups identified within the dynamic crowd, and the dynamic crowd as a whole entity.

The relevance of this research is that it derives a behavioural model of the dynamics of the crowds from empirical observation so this simulation will help to prevent crisis scenarios. Using and supporting simulations and visualization by efficient and ad-hoc data management makes it possible to address real time dynamic crowd observation and organization.

Decision making can consider the state of the dynamic crowd, the way it occupies space and the way individuals form or leave groups. With new technology it might be possible to configure and reconfigure urban spaces dynamically in order to prevent disasters and to help people to behave safely in collective events. These issues are part of our future work. Our future research will also seek to further identify the critical behaviour of an individual according to the group she belongs to and its characteristics. We also want to determine how to prevent the death of people through proper and efficient decision making processes using optimal humanitarian logistics.

\section{Acknowledgements}

This work has been partially funded by the CNRS through its UMI LAFMIA and the project CONCERNS which is partially supported by Microsoft Research via an Azure Data Science Grant.

\section{References}

[1] B. D. Hankin and R. A. Wright, "Passenger Flow in Subways," Operational Research Society, vol. 9, no. 2, p. 81, Jun. 1958.

[2] S. Older, "Movement of Pedestrians on Footways in Shopping Streets," Traffic Engineering \& Control, vol. 10, no. 4, pp. 160-163, 1968.

[3] U. Weidmann, "Transporttechnik der Fussgänger," Schriftenreihe des Instituts für Verkehrsplanung, Transporttechnik, Straßen- und Eisenbahnbau,
1992.

[4] J. J. Fruin, "Designing for pedestrians: A level-ofservice concept," in Highways Research Record Number 355: Pedestrians, Highway Research Board, 1971, pp. 1-15.

[5] J. Berclaz, F. Fleuret, and P. Fua, "Robust People Tracking with Global Trajectory Optimization," in Proc. of the IEEE Computer Society Conference on Computer Vision and Pattern Recognition (CVPR'06), 2006, vol. 1, pp. 744-750.

[6] K. Bolay, "Nichtlineare Phänomene in einem fluiddynamischen Verkehrsmodell," Master thesis, University of Stuttgart, 1998.

[7] A. Borgers and H. J. P. Timmermans, "City centre entry points, store location patterns and pedestrian route choice behaviour: A microlevel simulation model," Socio-Economic Planning Sciences, vol. 20, no. 1, pp. 25-31, 1986.

[8] V. Blue and J. Adler, "Emergent Fundamental Pedestrian Flows from Cellular Automata Microsimulation," Transportation Research Record: Journal of the Transportation Research Board, vol. 1644, pp. 29-36, Jan. 1998.

[9] TRM Transportation Research Board, Highway Capacity Manual (Special Report, 209). Washington, D.C, USA, 1994

[10] J. P. Keating, "The myth of panic," Fire, vol. 76, no. 3, pp. 57-61, 1982.

[11] D. Elliott and D. Smith, "Football stadia disasters in the United Kingdom: learning from tragedy?," Organization \& Environment, vol. 7, no. 3, pp. 205-229, Jan. 1993.

[12] D. J. Parker and J. Handmer, "Disaster at Hillsborough Stadium: A comparative analysis," in Hazard management and emergency planning: perspectives on Britain, James \& James, 1992.

[13] D. Caner, "Fires and human behaviour," 1990.

[14] Tao Zhao and R. Nevatia, "Bayesian human segmentation in crowded situations," in Proc. of the IEEE Computer Society Conference on Computer Vision and Pattern Recognition, 2003. Proceedings., 2003, vol. 2, pp. 459-466.

[15] M. Rodriguez, S. Ali, and T. Kanade, "Tracking in unstructured crowded scenes," in Proc. of the IEEE 12th International Conference on Computer Vision, 2009, pp. 1389-1396.

[16] G. J. Brostow and R. Cipolla, "Unsupervised Bayesian Detection of Independent Motion in Crowds," in Proc. of the IEEE Computer Society Conference on Computer Vision and Pattern Recognition (CVPR'06), 2006, vol. 1, pp. 594-601.

[17] S. Ali and M. Shah, "A Lagrangian Particle Dynamics Approach for Crowd Flow Segmentation and Stability Analysis," in Proc. of the IEEE Conference on Computer Vision and Pattern 
Recognition, 2007, pp. 1-6.

[18] N. Hu, H. Bouma, and M. Worring, "Tracking individuals in surveillance video of a high-density crowd," Proc SPIE, vol. 8399, May 2012.

[19] H. Jiang, S. Fels, and J. J. Little, "A Linear Programming Approach for Multiple Object Tracking," in Proc. of the IEEE Conference on Computer Vision and Pattern Recognition, 2007, pp. 1-8.

[20] C. Huang, B. Wu, and R. Nevatia, "Robust Object Tracking by Hierarchical Association of Detection Responses," in Computer Vision - ECCV 2008, Berlin, Heidelberg: Springer Berlin Heidelberg, pp. 788-801.

[21] B. Leibe, K. Schindler, and L. Van Gool, "Coupled Detection and Trajectory Estimation for MultiObject Tracking," in Proc. of the IEEE 11th International Conference on Computer Vision, 2007, pp. 1-8.

[22] Li Zhang, Yuan Li, and R. Nevatia, "Global data association for multi-object tracking using network flows," in Proc. of the IEEE Conference on Computer Vision and Pattern Recognition, 2008, pp. 1-8.

[23] Tao Zhao and R. Nevatia, "Tracking multiple humans in crowded environment," in Proc. of the IEEE Conference on Computer Vision and Pattern Recognition (CVPR'04), 2004, vol. 2, pp. 406-413.

[24] B. Wu and R. Nevatia, "Detection and Segmentation of Multiple, Partially Occluded Objects by Grouping, Merging, Assigning Part Detection Responses," International Journal of Computer Vision, vol. 82, no. 2, pp. 185-204, Apr. 2009.

[25] Yuan Li, Chang Huang, and R. Nevatia, "Learning to associate: HybridBoosted multi-target tracker for crowded scene," in Proc. of the IEEE Conference on Computer Vision and Pattern Recognition, 2009, pp. 2953-2960.

[26] Y. Li and H. Ai, "Fast Detection of Independent Motion in Crowds Guided by Supervised Learning," in Proc. of the IEEE International Conference on Image Processing, 2007.

[27] D. Sugimura, K. M. Kitani, T. Okabe, Y. Sato, and A. Sugimoto, "Using individuality to track individuals: Clustering individual trajectories in crowds using local appearance and frequency trait," in Proc. of the IEEE 12th International Conference on Computer Vision, 2009, pp. 14671474.

[28] H. Tsutsui, J. Miura, and Y. Shirai, "Optical flowbased person tracking by multiple cameras," in Proc. of the International Conference on Multisensor Fusion and Integration for Intelligent Systems, 2001, pp. 91-96.
[29] T. Yamane, Y. Shirai, and J. Miura, "Person tracking by integrating optical flow and uniform brightness regions," in Proc. of the IEEE International Conference on Robotics and Automation, 1998, vol. 4, pp. 3267-3272.

[30] L. Kratz and K. Nishino, "Tracking with local spatio-temporal motion patterns in extremely crowded scenes," in Proc. of the IEEE Conference on Computer Vision and Pattern Recognition, 2010, pp. 693-700.

[31] D. Helbing and P. Molnár, "Social force model for pedestrian dynamics," Physical Review E, vol. 51, no. 5, pp. 4282-4286, May 1995.

[32] D. Helbing, P. Molnár, I. J. Farkas, and K. Bolay, "Self-Organizing Pedestrian Movement," Environment and Planning B: Planning and Design, vol. 28, no. 3, pp. 361-383, Jun. 2001.

[33] S. J. Yuhaski and J. M. Smith, "Modeling circulation systems in buildings using state dependent queueing models," Queueing Systems, vol. 4, no. 4, pp. 319-338, Dec. 1989.

[34] D. Garbrecht, "Describing pedestrian and car trips by transition matrices," Traffic Quarterly, vol. 27, no. 1, pp. 89-109, 1973.

[35] N. Ashford, M. O'Leary, and P. D. McGinity, "Stochastic modelling of passenger and baggage flows through an airport terminal," Traffic Engineering \& Control, vol. 17, no. 5, pp. 207-210, 1976.

[36] D. Helbing, "Stochastische Methoden, nichtlineare Dynamik und quantitative Modelle sozialer Prozesse," PhD thesis, University of Stuttgart, 1996.

[37] L. F. Henderson, "On the fluid mechanics of human crowd motion," Transportation Research, vol. 8, no. 6, pp. 509-515, Dec. 1974

[38] R. L. Hughes, "A continuum theory for the flow of pedestrians," Transportation Research Part B: Methodological, vol. 36, no. 6, pp. 507-535, Jul. 2002.

[39] D. Helbing, "A Mathematical Model for the Behavior of Pedestrians," Behavioral Science, vol. 31, no. 1, pp. 298-310, May 1991.

[40] P. G. Gipps and B. Marksjö, "A micro-simulation model for pedestrian flows," Mathematics and Computers in Simulation, vol. 27, no. 2-3, pp. 95105, Apr. 1985.

[41] M. Fukui and Y. Ishibashi, "Self-Organized Phase Transitions in Cellular Automaton Models for Pedestrians," Journal of the Physical Society of Japan, vol. 68, no. 8, pp. 2861-2863, Aug. 1999.

[42] M. Muramatsu, T. Irie, and T. Nagatani, "Jamming transition in pedestrian counter flow," Physica A: Statistical Mechanics and its Applications, vol. 267, no. 3-4, pp. 487-498, May 1999. 
[43] H. Klüpfel, T. Meyer-König, J. Wahle, and M. Schreckenberg, "Microscopic Simulation of Evacuation Processes on Passenger Ships," in Theory and Practical Issues on Cellular Automata, Springer, 2001, pp. 63-71.

[44] C. Burstedde, K. Klauck, A. Schadschneider, and J. Zittartz, "Simulation of pedestrian dynamics using a two-dimensional cellular automaton," Physica A: Statistical Mechanics and its Applications, vol. 295, no. 3-4, pp. 507-525, Jun. 2001.

[45] S. Gopal and T. R. Smith, "NAVIGATOR: An Albased model of human way-finding in an urban environment," in Spatial Choices and Processes, Elsevier, 1990, pp. 169-200.

[46] C. W. Reynolds, "Evolution of Corridor Following Behavior in a Noisy World," in Proc.of the 3rd International Conference on Simulation of Adaptive Behavior (SAB94), 1994, pp. 402-410.

[47] C. W. Reynolds, "Flocks, herds and schools: A distributed behavioral model," in Proc. of the 14th annual conference on Computer graphics and interactive techniques (SIGGRAPH '87), 1987, pp. 25-34.

[48] S. Paris, J. Pettré, and S. Donikian, "Pedestrian Reactive Navigation for Crowd Simulation: a Predictive Approach," Computer Graphics Forum, vol. 26, no. 3, pp. 665-674, Sep. 2007.

[49] J. van den Berg, S. Patil, J. Sewall, D. Manocha, and M. Lin, "Interactive navigation of multiple agents in crowded environments," in Proc. of the 2008 Symposium on Interactive 3D Graphics and Games (S/3D'08), 2008, p. 139.

[50] J. van den Berg, S. J. Guy, M. Lin, and D. Manocha, "Reciprocal n-Body Collision Avoidance," in Robotics Research, Springer, 2011, pp. 3-19.

[51] J. Klockgether and H. P. Schwefel, "Two-phase nozzle and hollow core jet experiments," in Proc. of the 11th Symposium on Engineering Aspects of Magnetohydrodynamics., 1970, pp. 141-148.

[52] N. E. Miller, "Experimental studies of conflict behavior," Personality and behavior disorders, pp. 431-465, 1944.

[53] N. E. Miller, "Liberalization of basic S-R concepts: Extensions to conflict behavior, motivation and social learning," Psychology: A study of a science, Study 1, vol. 2, pp. 196-292, 1959.

[54] K. Lewin, Field theory in social science. Harper Torchbooks, 1964.

[55] D. Helbing, "A Mathematical Model for the Behavior of Individuals in a Social Field," Mathematical Sociology, vol. 19, no. 3, pp. 189219, May 1994.

[56] Yang, Gonzalez-Banos, and Guibas, "Counting people in crowds with a real-time network of simple image sensors," in Proc. of the 9th IEEE International Conference on Computer Vision, 2003, pp. 122-129.

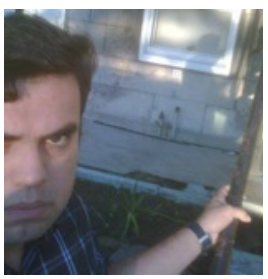

Carlos Alberto Ochoa OrtizZezzatti (Bs'94-Eng.Master'00; PhD'04-Postdoctoral Researcher'06 \& Industrial Postdoctoral Research'09). He joined the Juarez City University in 2008. He participates in the organization of several International Conferences. He has reviewed four important Journals from Elsevier: highlighting Applied Soft Computing and Computers in Human Behaviour. His research interests include ubiquitous computing, evolutionary computation, natural processing language, social modelling, anthropometrics characterization and Social Data Mining. In May 2016 he began a Sabbatical internship at the Barcelona Supercomputing Centre with a project related with simulation of human stampedes and refugee boat sinking improved with Artificial Intelligence. Actually he is member of the Mexican National System of Scientists of the CONACyT (SNI Level II).

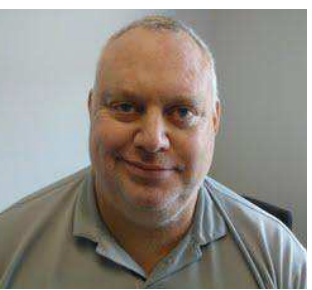

Isaac Rudomín (PhD'90-CS UPENN). He worked at Tecnológico de Monterrey Estado de Mexico Campus (ITESM-CEM) from 1991 to 2012, building Mexico's strongest group in computer graphics, working in human and crowd animation among other subjects. He started programming using the GPU in 2005, which led to his interest in HPC. In 2012 he joined the Barcelona Supercomputing Center. His research interests are in crowd simulation, generation and visualization in heterogeneous HPC systems.

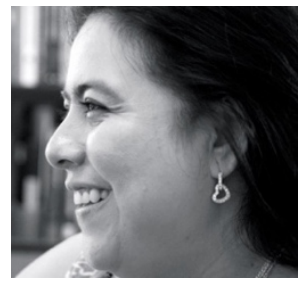

Genoveva Vargas-Solar received her first PhD on Computer Science from University Joseph Fourier and her second PhD from University Stendhal. She obtained her Habilitation à Diriger des Recherches (HDR tenure) from University of Grenoble. Her research interests in Computer Science concern distributed and heterogeneous databases, reflexive systems and service based database systems. She contributes to the construction of service based database management systems. She conducts fundamental and applied research activities for addressing these challenges on different architectures 
ARM, raspberry, cluster, cloud, and HPC. She has applied her results to e-Science applications in Astronomy, Biology, social sciences, industry 4.0.

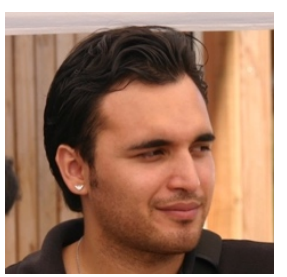

Javier A. Espinosa-Oviedo is a postdoctoral research fellow at Barcelona Supercomputing Centre (BSC) and member of the French-Mexican Laboratory of Informatics and Automatic Control (LAFMIA). He holds a $\mathrm{PhD}$ in Computer Science from University of Grenoble, France. His research concerns databases and distributed systems. In particular, he is interested on Internet Technologies (e.g., ServiceOriented Architectures, Cloud Computing, Data Services) and NoSQL solutions for modern data management. His objective is to design data management services guided by QoS criteria (e.g., security, reliability, fault tolerance, evolution and dynamic adaptability) and behavioural properties (e.g., transactional execution). He has participated in several national and international projects, where he has been responsible of the execution of working packages and the implementation of prototypes (POLIWEB PEPS CNRS; CASES EU-FP7; S2EUNET FP7-IRSES).

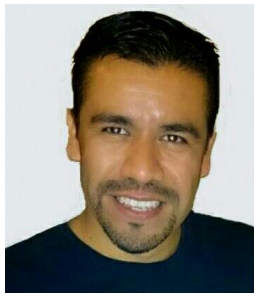

Hugo Pérez got his B.S. degree in Electronic Engineering from $\mathrm{Na}$ tional University in Mexico (UNAM). $\mathrm{He}$ got his M.Sc. degree in Computers Architecture, Networks and Systems from Universitat Politècnica de Catalunya BarcelonaTech. Currently he is working in the Parallel Programming Models Group at the Barcelona Supercomputing Centre as PhD student. His research project entitled "Crowd Simulation and Visualization" which aims to represent the most realistic possible scenarios in a city, these kind of systems allow: urban planning, simulating disasters, simulate epidemics, among other applications. The project combines different areas research such as: Big Data, Al, Parallel Programming Models, HPC, Computer Graphics, $\mathrm{HCl}$ between others.

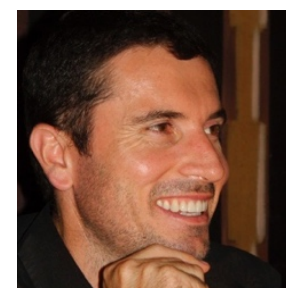

José-Luis Zechinelli-Martini is Associate professor of the Department of Computing, Electronics and Mecatronics at the Universidad de las Américas Puebla (UDLAP) since 2002 and he is currently senior researcher at LAFMIA. He is head of the Data and Knowledge Management Group (DBKM) of
LAFMIA. His fundamental research project addresses distributed databases on heterogeneous networks. The objective is to provide data access, querying and analysis adapted to the execution context by maximizing heterogeneous computing resources of continuous data production environments. He has developed his research through projects financed by governmental agencies like CUDI (project e-GrOV), the program ECOS-ANUIES, the LACCIR Microsoft program and the FP7 program of the European Community (projects S2EUNET and CASES).

Article received on 21/11/2011; accepted 21/11/2012. 